$51 \mid 2020$

Ladakh Through the Ages. A Volume on Art History and Archaeology, followed by Varia

Jahoda Christian, Socio-economic Organisation in a Border Area of Tibetan Culture, Tabo, Spiti Valley, Himachal Pradesh, India

Vienne, Verlag der österreichischen Akademie der Wissenschaften, 2015, 388 pages, ISBN 978-3-7001-7816-6

Patrick Kaplanian

\title{
OpenEdition
}

Journals

Édition électronique

URL : https://journals.openedition.org/emscat/4811

DOI : 10.4000/emscat.4811

ISSN : 2101-0013

Éditeur

Centre d'Etudes Mongoles \& Sibériennes / École Pratique des Hautes Études

Référence électronique

Patrick Kaplanian, « Jahoda Christian, Socio-economic Organisation in a Border Area of Tibetan Culture,

Tabo, Spiti Valley, Himachal Pradesh, India », Études mongoles et sibériennes, centrasiatiques et tibétaines [En ligne], 51 | 2020, mis en ligne le 09 décembre 2020, consulté le 13 juillet 2021. URL : http:// journals.openedition.org/emscat/4811 ; DOI : https://doi.org/10.4000/emscat.4811

Ce document a été généré automatiquement le 13 juillet 2021.

(c) Tous droits réservés 


\section{Jahoda Christian, Socio-economic Organisation in a Border Area of Tibetan Culture, Tabo, Spiti Valley, Himachal Pradesh, India}

Vienne, Verlag der österreichischen Akademie der Wissenschaften, 2015, 388 pages, ISBN 978-3-7001-7816-6

\section{Patrick Kaplanian}

\section{RÉFÉRENCE}

Jahoda Christian, Socio-economic Organisation in a Border Area of Tibetan Culture, Tabo, Spiti Valley, Himachal Pradesh, India, Vienne, Verlag der österreichischen Akademie der Wissenschaften, 2015

1 Le Ladakh fut ouvert aux étrangers en septembre 1974. Cela provoqua une ruée de chercheurs organisés aujourd'hui dans une International Association for Ladakh Studies (IALS).

2 Le Spiti, une autre région de l'Ouest himalayen de culture tibétaine, ne fut ouvert qu'en 1992 (p. 19). Le livre de Jahoda permet aux recherches sur le Spiti de rattraper une grande partie de leur retard.

3 Le livre n'est pas une monographie au sens traditionnel comme il y en a eu sur le Ladakh et il n'est donc pas exhaustif. Certains sujets comme la religion populaire ou les rituels sont à peine évoqués. Mais il dépasse largement le cadre de son titre très modeste, ce qui pourra déjà permettre de fructueuses comparaisons.

4 L'auteur distingue soigneusement deux régions qu'il étudie également. Le Spiti proprement dit, c'est-à-dire la vallée de la rivière du même nom qui se jette dans la Sutlej après être passée dans des gorges, et le Haut-Kinnaur, lui aussi bouddhiste et 
tibétophone, qui correspond au premier secteur de la vallée de la Sutlej après que ce fleuve a franchi la frontière du Tibet. Plus en aval c'est le Royaume de Bashahr. Cette séparation entre deux régions tibétophones et bouddhistes mitoyennes s'explique par des raisons historiques. Après la fin de la guerre tibéto-ladakho-moghole (1673-1683) le Spiti fut cédé au Tibet mais bien vite récupéré par le Ladakh, tandis que le HautKinnaur alla au royaume de Bashahr en remerciement de son soutien au Tibet contre le Ladakh (p. 57). Et les deux systèmes de gouvernement local étaient très différents. Même après l'arrivée des Anglais les deux systèmes (Spiti, p. 112 ; Haut-Kinnaur, p. 118) restèrent différents car les Britanniques avaient établi des protectorats qui s'appuyaient sur la hiérarchie sociale et l'organisation déjà en place.

5 Le livre se divise en trois grandes parties auxquelles il faut ajouter une copieuse documentation en annexe. Le premier chapitre, "History and geography ", de très loin le plus long (pp. 37-141), traite surtout d'histoire, depuis le royaume de Zhang Zhung au $\mathrm{VII}^{\mathrm{e}}$ siècle. Au début nous savons peu de choses sur le Spiti, mais nous sommes bien renseignés sur la fondation du royaume du Guge-Purang-Maryul (tib. mNga' ris skor gsum) par sKyid lde Nyi ma mgon, issu de la dynastie de Yarlung. Un de ses fils hérita peut-être du Spiti, mais les textes ne sont pas clairs et se contredisent (p. 44). Le petitfils de sKyid lde Nyi ma mgon se fit moine en 989 sous le nom de Ye shes'od, et son frère en fit autant en 996 (p. 54). C'est à cette époque-là que fut décidé qu'un certain nombre de maisonnées devaient payer une sorte d'impôt, non à l'État ou au souverain mais à un monastère pour l'entretien des moines. Ce système exista jusqu'au $\mathrm{xx}^{\mathrm{e}}$ siècle au Spiti, où une cinquantaine de fermes payaient pour l'entretien de cinq monastères, formant un groupement (l'auteur dit « district » mais les fermes ne sont pas groupées sur un territoire continu) et ne payaient pas d'impôt au roi. Comme le fait remarquer l'auteur lui-même nous sommes mieux renseignés sur la période qui va du $\mathrm{x}^{\mathrm{e}}$ au $\mathrm{XII}^{\mathrm{e}}$ siècle (p. 37) que sur la période qui va du XII siècle à l'arrivée des Anglais. Notons que le monastère de Tabo fut fondé en 996.

6 En 1396 la dynastie Namgyal prit le pouvoir puis ce fut la conversion de presque tous les monastères (y compris Tabo) à l'ordre gelukpa (tib. dge lugs pa). Le Spiti ne rentre véritablement dans l'histoire qu'après la défaite ladakhi contre le Tibet qui réoccupe mNga' ris skor gsum précédemment conquis par le roi ladakhi Sen ge rnam rgyal.

7 À partir de là, la lecture du livre devient un peu laborieuse. On a parfois l'impression que l'auteur publie ses notes de lecture les unes derrière les autres sans faire de synthèse. Prenons un exemple : le système de pouvoir après le retour du Spiti dans le giron ladakhi. Derrière une avalanche de citations partielles et apparemment contradictoires (pp. 59-60, 64-72, 73-78, 84-90, 111 et 118), le schéma est finalement très simple. Le Spiti est un district (ou waziri) dirigé par un wazir appartenant à la famille quasi-royale des Nono, il était divisé en cinq sous-districts appelés «kothi». Un de ces sous-district était appelé « sous-district religieux » (tib. chos gzhis). Il consistait en une cinquantaine de maisons qui, au lieu de payer un impôt au souverain (le roi du Ladakh, puis le raja dogra, puis l'autorité britannique), le payaient aux cinq principaux monastères. Cet «impôt " s'appelait «pun» (probablement «bon» en tibétain). Les quatre autres sous-districts, qui réunis ensemble formaient le "sous-district royal» (tib. khalsa khoti), payaient la "taxe en orge" (tib. nas khral), au pouvoir politique. Chaque sous-district était dirigé par un « chef de district » (tib. rgad po chen mo ou kothi rgad po), qui assistait le Nono. Et chaque village était dirigé par un rgad po chung ngung (au Ladakh on dit "go pa) qui percevait la " taxe en orge " (tib. nas khral). Comme le fait 
remarquer la Gazetteer of Kangra, 1883 (Punjab Government 1884, part. IV, Spiti, p. 76) : " the state of affairs described in Moorcroft and Gerard's Travels as existing nearly seventy years ago [...] are managed in much the same way at the present day ». Seules différences : le roi du Ladakh avait coiffé le tout d'un « chef du palais » (tib. mkhar dpon) et le souverain dogra d'un "chef de district " (thanadar, dans le vocabulaire propre aux Dogra). À noter aussi que les Britanniques eurent beaucoup de mal à comprendre le système du " sous-district religieux ", comme en témoignent les pages 112 à 118 de la partie IV de la Gazetteer of Kangra, 1883, (qui inclut le Spiti), publiée en 1884.

La troisième partie, intitulée «Peasantry and development » (pp. 143-158), résume le long chapitre précédent en divisant l'histoire en neuf phases. L'auteur passe un peu vite sur les dernières phases (phase VII, 1846-1947, pp.153-154; phase VIII, 1947-1975; phase IX, 1875 à aujourd'hui, toutes deux p. 158). On aurait aimé en savoir plus sur les conséquences des réformes agraires dont la liste est donnée page 132, note 238.

La quatrième partie intitulée " Tabo » (pp. 159-225), est la plus intéressante car elle est de première main, suite aux terrains de l'auteur. Elle décrit le monastère et le village de Tabo. Sont décrits la stratification sociale, la parenté, les règles d'héritage, l'organisation du village, le calendrier agricole, etc.

Il ressort de cette très riche description que Tabo est un village atypique. Ainsi Jahoda écrit-il (p.190) qu'il a remarqué 10 à $20 \%$ de polyandrie à Tabo dans les "maisons principales " (tib. khang chen) alors que Tashi Tsering écrit que « another unique aspect of Spiti, is that it is rare to find polyandrous relations. In the two villages where I conducted this study, there was only one household with polyandrous arrangement " (Tashi Tsering 2014, p. 50, n. 31). Ce qui confirme ce qu'écrivait Lyall (1874) 150 ans plus tôt.

11 Mais ceci est un détail. La différence qu'il est important de souligner c'est qu'à Tabo il y a des "maisons principales", des "maisons annexes" mais pas de du 'thul. Ici il convient de rappeler quelques concepts. La khang chen est la maison principale, la khang chung est une maison annexe où se retirent les vieux parents. Au Ladakh tout comme dans les villages du Spiti décrits par Tashi Tsering les maisons annexes n'ont pas de personnalité juridique, pas de droits et de devoirs, pas d'existence propre. La maison annexe fait partie de la maison principale. Les $d u$ 'thul, déjà longuement décrits par Lyall au XIXe siècle, sont des petites maisons entourées de peu de terres, voire pas du tout, et dont les habitants survivaient comme serviteurs auprès des maisons principales, mais qui s'en sortent mieux depuis les distributions de terres qui ont suivi l'indépendance. Les $d u$ 'thul sont inconnus au Ladakh mais connus au Tibet où ils ont été décrits par Goldstein (1971) et par Aziz (1978) sous le nom de " dud chung ».

Donc dans les deux villages étudiés par Tashi Tsering les maisons annexes n'existent pas en tant que telles: «khangchung and yangchung [annexes des khangchung] households are thus extended residence of the khangchen households " (Tashi Tsering 2014, p. 54, n. 35) et "khangchung are technically part of the khangchen household " (ibid., pp. 121-122, n. 111). Par contre les $d u$ 'thul constituent la majorité de la population (ibid., p. 56). En 2001 au village de Zibug on comptait 13 maisons principales, $53 \mathrm{du}$ 'thul, 4 maisons de forgerons et 3 maisons de musiciens (ibid., p. 223). Et cette population est en forte augmentation à cause des réformes agraires. Ainsi, de 1968 à 1970, 37 champs ont été distribués à Zibug: 23 à des du 'thul et 14 à des forgerons. Que les maisons annexes ne soient pas séparées des maisons principales, cela ressort des règles de distribution de l'eau. Une part (la plus grosse) va aux maisons 
principales (maisons annexes comprises) et le reste aux $d u$ 'thul, aux forgerons et aux musiciens.

13 À Tabo il n'y a pas de $d u$ 'thul et les maisons annexes sont séparées des maisons principales. Il y a même en plus du «chef de village » (nambardar) un second chef de village propre aux maisons annexes (p.196). Et la répartition de l'eau est tout aussi inégale qu'à Zibug: 3 maisons annexes sont associées à une maison principale: la maison principale reçoit l'eau pour 24 ou $36 \mathrm{~h}$ et les 3 maisons annexes pour $12 \mathrm{~h}$ (p. 210). Mais cette répartition inégale se fait entre maisons principales et maisons annexes et non pas entre maisons principales et $d u$ 'thul.

Cette partie qui décrit Tabo est extrêmement riche. Ce qui fait de ce livre une véritable introduction au Spiti, incontournable pour toute personne qui s'intéresse à l'Himalaya de l'Ouest.

Enfin il faut dire quelques mots des très riches annexes: une centaine de pages; de nombreuses photos en couleurs illustrant le cycle agricole et le cycle rituel. Et des textes (photographies, translittérations et traductions), précieux documents permettant de mieux comprendre la vie à Tabo.

\section{BIBLIOGRAPHIE}

Aziz, B. M. 1978 Tibetan Frontier Families. Reflections on Three Generations of D'ing-ri (New Delhi, Vikas Publishing House).

Punjab Government 1884 Gazetteer of Kangra district, 1883, vol. 2, Kulu, Lahul \& Spiti (Calcutta, Central Press Company).

Goldstein, M. 1971 Taxation and the structure of a Tibetan village, Central Asiatic Journal 15(1), pp. 1-27.

Lyall, J. B. 1874 Report of the Land Revenue Settlement of the Kangra District, Panjab (Lahore, Central Jail Press).

Tashi Tsering 2014 Social Inequality and Resource Management. Gender, Caste and Class in the Rural Himalayas. Thèse de doctorat dactylographiée (Vancouver, The university of British Columbia). 